\title{
APPLYING THE PROPORTIONAL HAZARD PREMIUM CALCULATION PRINCIPLE
}

\author{
BY
}

Maria de Lourdes Centeno and João Andrade E Silva

\begin{abstract}
In this paper we discuss the application of the proportional hazard premium calculation principle.

In Section 2 we propose a method to calculate the proportional hazard premium of a compound risk when the severity distribution is subexponential.

In Section 3 we use the empirical distribution to calculate the premium when the proportional hazard principle is applied, which leads to a systematic underestimation of the premium. After studying the bias of the premium calculated using this non-parametric approach we take advantage of the bootstrap technique with subsampling to reduce it.
\end{abstract}

\section{KEYWORDS}

Proportional hazard premium principle, subexponential distributions, bootstrap, subsampling.

\section{INTRODUCTION}

As it is well known the proportional hazard premium principle ( $\mathrm{PH}$ premium principle), introduced by Wang (1995), satisfies properties which make of it a very attractive premium from the theoretical point of view, see e.g. Wang (1996) and Andrade e Silva and Centeno (1998). However its use depends on the complete knowledge of the distribution of the aggregate claims amount. In practical terms this means that one must fit a distribution to the data set or use the empirical distribution function. When the aggregate claims amount follows a compound distribution the calculation of the $\mathrm{PH}$ premium based on the parametric approach can raise some problems. This will be discussed in the following section.

The non-parametric approach is more appealing, from the practical point of view, but can lead to a significant underestimation of the premium. In subsection 3.1 we calculate the bias of the premium based on the empirical distribution for some distributions, namely the exponential, Pareto and uniform. We also study the rate of convergency of the bias in the exponential case. 
In subsection 3.2 we show how to use the bootstrap technique with subsampling to reduce the bias of the estimator of the premium based on the empirical distribution. We also perform two simulation studies to give some insight to this technique.

\section{APPLYING THE PH TRANSFORM TO COMPOUND DISTRIBUTIONS IN THE PARAMETRIC MODEL}

Let $Y$, the aggregate claims amount, be a nonnegative random variable, with distribution function $F(y)$. Let $S(y)=1-F(y)$ be the survival function. The PH premium principle assigns to the distribution $F(y)$ the premium

$$
\pi_{\rho}=\int_{0}^{\infty}(S(y))^{1 / \rho} d y
$$

where $\rho$, with $\rho \geq 1$, is risk aversion index.

When using the collective risk model, $Y$ is equal to $X_{1}+\ldots+X_{N}$, where $N$ is the number of claims occurred in a given period and $\left\{X_{i}\right\}_{i=1,2, \ldots}$ are the individual claims amounts which are assumed to be nonnegative i.i.d. random variables and independent of $N$. In this case $Y$ has a compound distribution and the company often estimates claim frequency and claim severity separately. Various numerical techniques are available to calculate the compound distribution, being Panjer's recursion formula the most well known. When using the recursion formula we will have to stop somewhere the calculations. When the individual claims amount has distribution $G$ with unlimited support, i.e. $G(x)<1$, for all $x>0$, and it is very skewed, the choice of the value where to stop the calculations to obtain a reasonable estimate of the premium is a critical aspect of the model. When $X$ follows a heavy tail distribution $\int_{t}^{\infty}(S(y))^{1 / \rho} d y$ can be of an expressive size even for large values of $t$. In this case, when the probability generating function of $N$ is analytic in the neighbourhood of 1 (which happens both for the Poisson and the negative binomial case) and $G$ is subexponential then (see Embrechts et al. (1997), pp. 45-46)

$$
1-F(x) \sim \mathrm{E}[N](1-G(x)), x \rightarrow \infty .
$$

Hence for heavy tail severity distributions we will approximate the premium by

$$
\pi_{\rho}^{*}=\int_{0}^{t}\left(S^{*}(y)\right)^{1 / \rho} d y+(\mathrm{E}[N])^{1 / \rho} \int_{t}^{\infty}(1-G(y))^{1 / \rho} d y,
$$

where $S^{*}(y)$ is the approximation to $S(y)$ obtained by Panjer's recursion formula, and $t$ is a suitable high value.

Example 1. Suppose that an actuary has arrived to the following estimates for a risk: the number of claims greater than a given observation point $d=100 \mathrm{~K}$ is Poisson distributed with mean $\lambda_{d}=6$ and the size of each claim greater than $d$ is Pareto with parameter $\alpha=1.647$. Let us consider an excess of loss reinsurance 
treaty. For a layer $l x s m$, with $m \geq d$ and $l \leq+\infty$, the aggregate claims amount are compound Poisson with expected number of claims $\lambda=(d / m)^{\alpha} \lambda_{d}$ and severity distribution

$$
G(x)= \begin{cases}1-\left(\frac{m}{m+x}\right)^{\alpha} & \text { if } 0 \leq x<l \\ 1 & \text { if } x \geq l\end{cases}
$$

Using $1 / \rho=0.9025$, Table 1 shows the $\mathrm{PH}$ transform premium, as percentage of the subject earned premium, $S E P=\$ 10,000 K$, associate to the following layers:
1. $\$ 400 K \mathrm{xs} \$ 100 K$
2. $\$ 500 K \mathrm{xs} \$ 500 K$
3. $\$ 900 K$ xs $\$ 100 K$
4. the excess over $\$ 1,000 \mathrm{~K}$

This example for the limited layers was considered by Wang (1998). For the calculation of the premium of the unlimited layer we have used (3) with $t=\$ 10^{5} \mathrm{~K}$, for which the difference between the aggregate survival function $S^{*}(t)$ and $\lambda(1-G(t))$, is $2.3 \times 10^{-7}$. The step used in the arithmetization of the severity distribution to perform the recursion was $h=\$ 1 K$.

The reason to chose such a high value for $t$ in formula (3) is related to the very small value of $\alpha$. If instead of $\$ 10^{5} \mathrm{~K}$ we had used $\$ 10^{4} \mathrm{~K}$ for $t$, the result of applying formula (3) with $\rho=1$ would be $2.086 \%$ of $S E P$, compared to a theoretical value of $2.090 \%$. Using $t=\$ 10^{5} \mathrm{~K}$ the two terms in the right hand side of (3) are $1.9848 \%$ and $0.1055 \%$ for $\rho=1$ and $3.0298 \%$ and $0.3580 \%$ for $1 / \rho=0.9025$. Note that the relative weight of the second term increases with $\rho$ and is far from negligible in spite of the high values of $t$.

\section{APPLYING THE PH TRANSFORM TO THE EMPIRICAL DISTRIBUTION}

\subsection{The bias of the premium}

Let $F_{n}(y)$ be the empirical distribution function of $Y$ based on a random sample of size $n,\left(Y_{1}, \ldots, Y_{n}\right)$, let $S_{n}(y)$ be the corresponding empirical survival function and

TABLE 1

PH-TRANSFORM PREMIUMS

\begin{tabular}{ccc}
\hline \hline Layer $l$ xs $m$ & $\begin{array}{c}\text { Pure Premium } \\
\text { as percentage of SEP }\end{array}$ & $\begin{array}{c}\pi_{\rho}^{*} \\
\text { as percentage of SEP }\end{array}$ \\
\hline$\$ 400 K$ xs $\$ 100 K$ & $6.000 \%$ & $6.384 \%$ \\
$\$ 500 K$ xs $\$ 500 K$ & $1.183 \%$ & $1.408 \%$ \\
$\$ 900 K$ xs $\$ 100 K$ & $7.183 \%$ & $7.742 \%$ \\
Xs $\$ 1,000 K$ & $2.090 \%$ & $3.388 \%$ \\
\hline \hline
\end{tabular}




$$
\hat{\pi}_{\rho}=\int_{0}^{\infty}\left(S_{n}(y)\right)^{1 / \rho} d y
$$

the premium estimator. Although $S_{n}(y)$ is an unbiased estimator of $S(y), \hat{\pi}_{\rho}$ given by (5) is a biased estimator of $\pi_{\rho}$, since

$$
E\left[\hat{\pi}_{\rho}\right]=\int_{0}^{\infty} E\left[\left(S_{n}(y)\right)^{1 / \rho}\right] d y \leq \int_{0}^{\infty}\left[E\left(S_{n}(y)\right)\right]^{1 / \rho} d y=\pi_{\rho}
$$

by Jensen's inequality, with the inequality being strict unless $\rho=1$.

Let $Y_{k: n}$ be the $k$-th order statistic in a sample of size $n$. Then

$$
S_{n}(y)=\left\{\begin{array}{cc}
1 & y<Y_{1: n} \\
(n-k) / n, & Y_{k: n} \leq y<Y_{k+1: n}, k=1, \ldots, n-1 \\
0 & y \geq Y_{n: n},
\end{array}\right.
$$

which implies that

$$
\hat{\pi}_{\rho}=\sum_{k=0}^{n-1}\left(\frac{n-k}{n}\right)^{1 / \rho}\left(Y_{k+1: n}-Y_{k: n}\right)
$$

where $Y_{0: n} \equiv 0$.

Let $F_{k: n}(y)$ be the distribution function of the $k$-th order statistic, i.e. $F_{k: n}(y)=$ $\operatorname{Pr}\left\{Y_{k: n} \leq y\right\}$. Given that

$$
F_{k: n}(y)=\sum_{r=k}^{n}\left(\begin{array}{l}
n \\
r
\end{array}\right)(F(y))^{r}(1-F(y))^{n-r},
$$

then

$$
1-F_{k: n}(y)=1-F_{k+1: n}(y)-\left(\begin{array}{l}
n \\
k
\end{array}\right)(F(y))^{k}(1-F(y))^{n-k}
$$

and integrating we obtain

$$
E\left[Y_{k+1: n}-Y_{k: n}\right]=\int_{0}^{\infty}\left(\begin{array}{l}
n \\
k
\end{array}\right)(F(y))^{k}(1-F(y))^{n-k} d y .
$$

Then, using (6) and (7) we get

$$
\begin{aligned}
E\left[\hat{\pi}_{\rho}\right] & =\sum_{k=0}^{n-1}\left(\frac{n-k}{n}\right)^{1 / \rho}\left(\begin{array}{l}
n \\
k
\end{array}\right) \int_{0}^{\infty}(F(y))^{k}(S(y))^{n-k} d y \\
& =\sum_{k=1}^{n}\left(\frac{n-k+1}{n}\right)^{1 / \rho}\left(\begin{array}{c}
n \\
k-1
\end{array}\right) \int_{0}^{\infty}(F(y))^{k-1}(S(y))^{n-k+1} d y
\end{aligned}
$$

which is, when $F$ is absolutely continuous for $x>0$, equivalent to

$$
E\left[\hat{\pi}_{\rho}\right]=\sum_{k=1}^{n}\left(\frac{n-k+1}{n}\right)^{1 / \rho}\left(\begin{array}{c}
n \\
k-1
\end{array}\right) \int_{0}^{1}(1-x)^{k-1} x^{n-k+1} \frac{1}{f\left[S^{-1}(x)\right]} d x,
$$


where $f(x)=F^{\prime}(x)$.

Expressions (8) and (9) can be used to calculate the bias of $\hat{\pi}_{\rho}$

$$
B\left(\hat{\pi}_{\rho}\right)=E\left[\hat{\pi}_{\rho}\right]-\pi_{\rho} .
$$

For some distributions (not in the compound case) the bias is easily calculated, as it is the case when $Y$ is exponential, Pareto or uniform, as can be seen in the following examples.

Example 2. Let $Y$ be exponential distributed, i.e. $S(y)=\exp (-\theta y), y>0(\theta>0)$. In this case $f\left[S^{-1}(x)\right]=\theta x$, and using (9) we get, after some calculations,

$$
\mathrm{E}\left[\hat{\pi}_{\rho}\right]=n^{-1 / \rho} \theta^{-1} \sum_{k=1}^{n} k^{1 / \rho-1}
$$

and

$$
B\left(\hat{\pi}_{\rho}\right)=\left(n^{-1 / \rho} \sum_{k=1}^{n} k^{1 / \rho-1}-\rho\right) \theta^{-1}
$$

Example 3. Let $Y$ be Pareto distributed, i.e. $S(y)=\left(\frac{\beta}{\beta+y}\right)^{\alpha}, y>0(\alpha, \beta>0)$. In this case $f\left[S^{-1}(x)\right]=\alpha \beta^{-1} x^{1+1 / \alpha}$, and using (9) we get,

$$
\mathrm{E}\left[\hat{\pi}_{\rho}\right]=\beta \alpha^{-1} n^{-1 / \rho} n ! \sum_{k=1}^{n} k^{1 / \rho} \frac{\Gamma(k-1 / \alpha)}{k ! \Gamma(n+1-1 / \alpha)}
$$

and

$$
B\left(\hat{\pi}_{\rho}\right)=\beta\left[\frac{n !}{\alpha n^{1 / \rho}} \sum_{k=1}^{n} k^{1 / \rho} \frac{\Gamma(k-1 / \alpha)}{k ! \Gamma(n+1-1 / \alpha)}-\frac{\rho}{\alpha-\rho}\right] .
$$

Example 4. When $Y$ is uniformly distributed in $(0,1)$ we get

$$
\mathrm{E}\left[\hat{\pi}_{\rho}\right]=\frac{1}{(n+1) n^{1 / \rho}} \sum_{k=1}^{n} k^{1 / \rho}
$$

and

$$
B\left(\hat{\pi}_{\rho}\right)=\frac{1}{(n+1) n^{1 / \rho}} \sum_{k=1}^{n} k^{1 / \rho}-\frac{\rho}{\rho+1} .
$$

For the exponential distribution it is possible to relate the order of the bias with the sample size, as stated in the result that follows. We were not able of developing similar results for other distributions, but we are strongly convinced that the order of the bias is smaller for the uniform and bigger for the Pareto.

Result. When $Y$ follows an exponential distribution $\left|B\left(\hat{\pi}_{\rho}\right)\right|$ converges to zero at the same rate as $n^{-1 / \rho}$. 
Proof. Noticing that

$$
\rho=n^{-1 / \rho} \sum_{k=1}^{n} \int_{0}^{1}(k-x)^{1 / \rho-1} d x
$$

and using (10) we have

$$
\left|B\left(\hat{\pi}_{\rho}\right)\right|=\theta^{-1} n^{-1 / \rho} \sum_{k=1}^{n} \int_{0}^{1}\left[(k-x)^{1 / \rho-1}-k^{1 / \rho-1}\right] d x .
$$

As $(k-x)^{1 / \rho-1}$ is an increasing function of $x$ for $k \geq 1$ and $0<x<1$, we have that

$$
\begin{gathered}
\sum_{k=1}^{n} \int_{0}^{1}\left[(k-x)^{1 / \rho-1}-k^{1 / \rho-1}\right] d x \leq \int_{0}^{1}\left[(1-x)^{1 / \rho-1}-1\right] d x \\
+\sum_{k=2}^{n}\left[(k-1)^{1 / \rho-1}-k^{1 / \rho-1}\right]=\rho-n^{1 / \rho-1} \leq \rho .
\end{gathered}
$$

On the other hand as $\int_{0}^{1}\left[(k-x)^{1 / \rho-1}-k^{1 / \rho-1}\right] d x$ is strictly positive we can conclude that the left hand side of (12) converges to a positive value $A$ as $n$ goes to infinity. Consequently

$$
\lim _{n \rightarrow \infty} n^{1 / \rho}\left|B\left(\hat{\pi}_{\rho}\right)\right|=\theta^{-1} A
$$

\subsection{Correcting the bias via bootstrapping}

As we have seen, the distortion of the empirical distribution leads to an underestimation of the premiums. We use the bootstrap technique, see e.g. Efron and Tibshirani (1993), to correct, at least partially, the bias of that estimator.

The bootstrap technique consists, as it is well known, on the resampling of the original data set. The bootstrap estimator of the bias of $\hat{\pi}_{\rho}$, is

$$
\widehat{B\left(\hat{\pi}_{\rho}\right)}=\hat{\pi}_{\rho}^{*}-\hat{\pi}_{\rho}
$$

with

$$
\hat{\pi}_{\rho}^{*}=\frac{1}{M} \sum_{b=1}^{M} \hat{\pi}_{\rho}^{{ }^{*} b}
$$

where $M$ is the number of bootstrap samples and $\hat{\pi}_{\rho}^{* b}$ is calculated using the $b$ th bootstrap sample. Hence the bootstrap estimator of the premium $\pi_{\rho}$ is

$$
\hat{\hat{\pi}}_{\rho}=\hat{\pi}_{\rho}-\widehat{B\left(\hat{\pi}_{\rho}\right)} \text {. }
$$

Usually the resampling is made with replacement and using bootstrap samples of size $n$ (i.e. the same size as the original sample). However in this case, as 


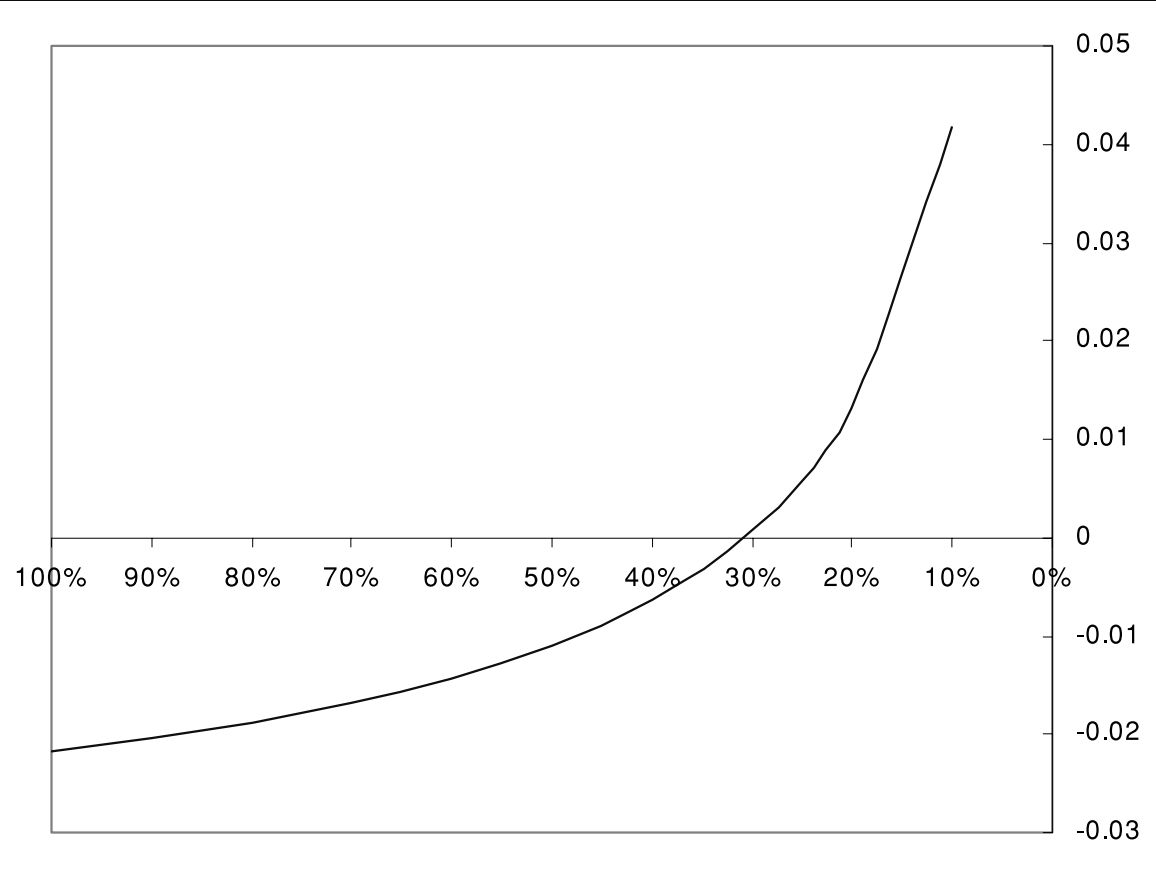

FIGURE 1: $\overline{B\left(\hat{\hat{\pi}}_{\rho}\right)}$ as function of $n^{\prime} / n$

$B\left(\hat{\pi}_{\rho}\right)$ is always negative, we can improve, in principle, the results by using bootstrap samples of size $n^{\prime}<n$. The reason for the bias to be negative is the underestimation of the right tail of the distorted distribution. The use of a subsampling in the bootstrap method intends to replicate that behaviour and consequently to obtain a better bias correction than the one obtained with the full sample.

Figure 1 shows how the bias of the corrected estimator given by (3) varies with the sample size $n^{\prime}$ (measured as a percentage of $n$ ). The figure has been drawn using a simulation approach (see simulation 1 below) for a Pareto distribution with $\alpha=4$, sample size of 500 observations, $\rho=1.2$ and using 2000 replicas and 2000 bootstraps samples for each replica. The shape of the curve is very similar for all the other situations: it starts, when $n^{\prime}=n$, with a negative value for the mean of the observed bias of the bootstrap premiums, which we denote $\overline{B\left(\hat{\hat{\pi}}_{\rho}\right)}$, then there is an optimal value of $n^{\prime}$ where $\overline{B\left(\hat{\hat{\pi}}_{\rho}\right)}$, is almost zero, and becomes positive when $n^{\prime}$ is very small. The question is how to choose $n^{\prime}$, independently of the distribution.

To give some insight to the problem we performed two simulation studies. In these studies we also use the Jackknife, as an alternative to the bootstrap, to correct the bias. As we will see the Jackknife performs better than full sampling bootstrap, but we can do better by using a suitable level of subsampling. 
Simulation 1. This case is based on two families of distributions, the Pareto and the Gamma. In the Pareto case we have considered that the parameter $\alpha$ takes the value 2, 3 or 4, originating what we call $\mathrm{Pareto}_{2}, \mathrm{Pareto}_{3}$ and Pareto respectively. The other parameter was chosen such that the distribution has mean equal to 1, i.e. we have assumed that the survival function for the Pareto ${ }_{\alpha}$ is

$$
S_{\alpha}(x)=\left(\frac{\alpha-1}{x+\alpha-1}\right)^{\alpha}, \quad x>0 .
$$

For the gamma distribution we have kept the same mean and assumed that the variance was equal to 1 (exponential) or 2 . We have considered that the original sample size is either 100,500 or 1000 . Tables 2 and 3 give the bootstrap and Jackknife results for $\rho=1.2$ and $\rho=1.15$ respectively, with $M=2000$ and using 2000 replicas.

As we have mentioned, the bias of the premium based on the empirical distribution (column labelled $B\left(\hat{\pi}_{\rho}\right)$, and calculated using expressions (8), (10) and (11), for the gamma, exponential and Pareto distributions) can be of a considerable size, namely for heavy tail distributions when $n$ is small and $\rho$ is high. For instance for the Pareto ${ }_{2}$, with $n=100$ and $\rho=1.2$, the absolute value of the bias is $8.4 \%$ of the theoretical premium. The average bias of the premiums based on the 2000 replicas (before the bootstrap is performed), labelled $\bar{B}$, is in those cases still a bit far from $B\left(\hat{\pi}_{\rho}\right)$, but it was out of our computing facilities to consider a big enough number of replicas in the simulation study. The following 10 columns show the average bias for the bootstrap premiums. The bootstrap with $n^{\prime}=n$, only corrects the bias partially, since after that correction we still observe a negative bias in all the situations of our example. As we can see from Tables 2 and 3, the pattern shown in Figure 1 is characteristic of the behaviour of the bias as function of $n^{\prime} / n$. The optimal proportion $n^{\prime} / n$ depends, of course, on the distribution, on $n$ and on $\rho$. As a rule $n^{\prime} / n$ should increase with $n$ and decrease with $\rho$. For instance, for $\rho=1.2$, a bootstrap with a resampling of $n^{\prime} / n=40 \%$ performs, in average, better than the bootstrap with full resampling (and certainly better than with no bootstrap), at least for the sample sizes considered in the study. The following column, labelled $J N$, shows the average bias for the Jackknife premiums, i.e. we use the Jackknife technique instead of bootstrap to correct the bias of the premiums. As we can see the Jackknife performs better than the full sampling bootstrap but, using subsampling, we can obtain a smaller bias with the bootstrap.

The last column of the tables, provides the average bias of the premiums if we have used the maximum likelihood estimators of the parameters. For the Pareto $_{2}$, the figures are not presented because, for a large number of samples ( 20 for $\rho=1.2$ and 11 for $\rho=1.15$ ), we got a maximum likelihood estimate $\hat{\alpha}$ smaller than $\rho$. In some other situations the estimate was very close to $\rho$, which would imply an extremely high premium. For the $\mathrm{Pareto}_{3}$ and $\mathrm{Pareto}_{4}$ we didn't get any sample with a maximum likelihood estimate smaller than $\rho$, but we got values not very far from $\rho$, which explain the positive values of the bias in those cases. The maximum likelihood performs well (in association with 
the $\mathrm{PH}$ premium calculation principle) if the distribution does not have a very heavy tail, or if the sample size is big enough.

As a conclusion, and for values of $\rho$ around 1.15-1.2 we can say that use of the bootstrap technique with a resampling proportion size of $40 \%$ of the original sample size provides good results in general, but when we know that we are dealing with heavy tail loss distributions, we could use a smaller resampling size.

Table 4 is equivalent to Table 2 but with $M=5000$. As it can be seen, the results are quite similar. Hence $M=2000$ seems enough.

Simulation 2. The goal of the second simulation study is to analyse the behaviour of our procedures when the aggregate claims amount are generated according to a compound Poisson or negative binomial distributions. For the individual claims amount we have considered the three Pareto's and the exponential distributions of the former example. The expected number of claims was set at 1 . As in the previous simulation study $\rho=1.2$ and $\rho=1.15, M=2000$, we use 2000 replicas and $n$ is equal to 100,500 or 1000 .

Tables 5 and 6 refer to the compound Poisson case and are similar to tables 2 and 3 , with the exceptions of $B\left(\hat{\pi}_{\rho}\right)$ and ML, which are not considered now. Column labelled $\pi_{\rho}^{*}$ was obtained using (3), with $t$ equal to 10000, 926 and 297 when $\alpha$ equal to 2, 3 and 4 respectively, values for which the $\operatorname{Pr}\{X>t\}=10^{-8}$. For the exponential case we only considered the first term of (3), with $t=9000$.

As expected with $\lambda=1$ the premiums associated to the compound distribution are greater than for the corresponding Pareto's.

The results are very similar to the former case. We obtained the same behaviour for the bias as a function of $n^{\prime} / n$, and again we would recommend a resampling of $n^{\prime} / n=40 \%$, but if we knew that we were dealing with heavy tail loss distributions, we could use a smaller resampling size.

The results for the simulations with $M=5000$ give very similar values, reason why we do not provide them here.

Tables 7 and 8 refer to the negative binomial case, with $\rho=1.2$ and with the variance of the number of claims equal to 1.05 and 1.2 respectively. As we can see the results lead to the same conclusion.

\section{ACKNOWLEDGMENTS}

This research was supported by Fundação para a Ciência e a Tecnologia - FCT/ POCTI. We are also grateful to our colleague Raul Brás for assisting us with the computation programs. We also thank an anonymous referee for several suggestions which helped to improve the paper.

\section{REFERENCES}

Andrade e Silva, J. and Centeno, M.L. (1998) Comparing risk adjusted premiums from the reinsurance point of view. ASTIN Bulletin, 28, 221-239. 
Embrechts, P., KlüPpelberg C., Mikosch, T. (1997) Modelling extremal events. Springer-Verlag, Berlin.

Efron, B. and Tibshirani, R. (1993) An Introduction to the Bootstrap. Chapman and Hall, London.

WANG, S. (1995) Insurance pricing and increased limits ratemaking by proportional hazards transforms. Insurance: Mathematics and Economics, 17, 43-54.

Wang, S. (1996) Premium calculation by transforming the layer premium density, ASTIN Bulletin, 26, 71-92.

WANG, S. (1998) Implementation of PH-transforms in ratemaking, Proceedings of the Casualty Actuarial Society, LXXXV, 940-979.

\section{Maria de Lourdes CENTENo \\ CEMAPRE, ISEG, \\ Technical University of Lisbon,}

Rua do Quelhas, 2, 1200781 Lisbon,

Portugal

E-mail:lcenteno@iseg.utl.pt 


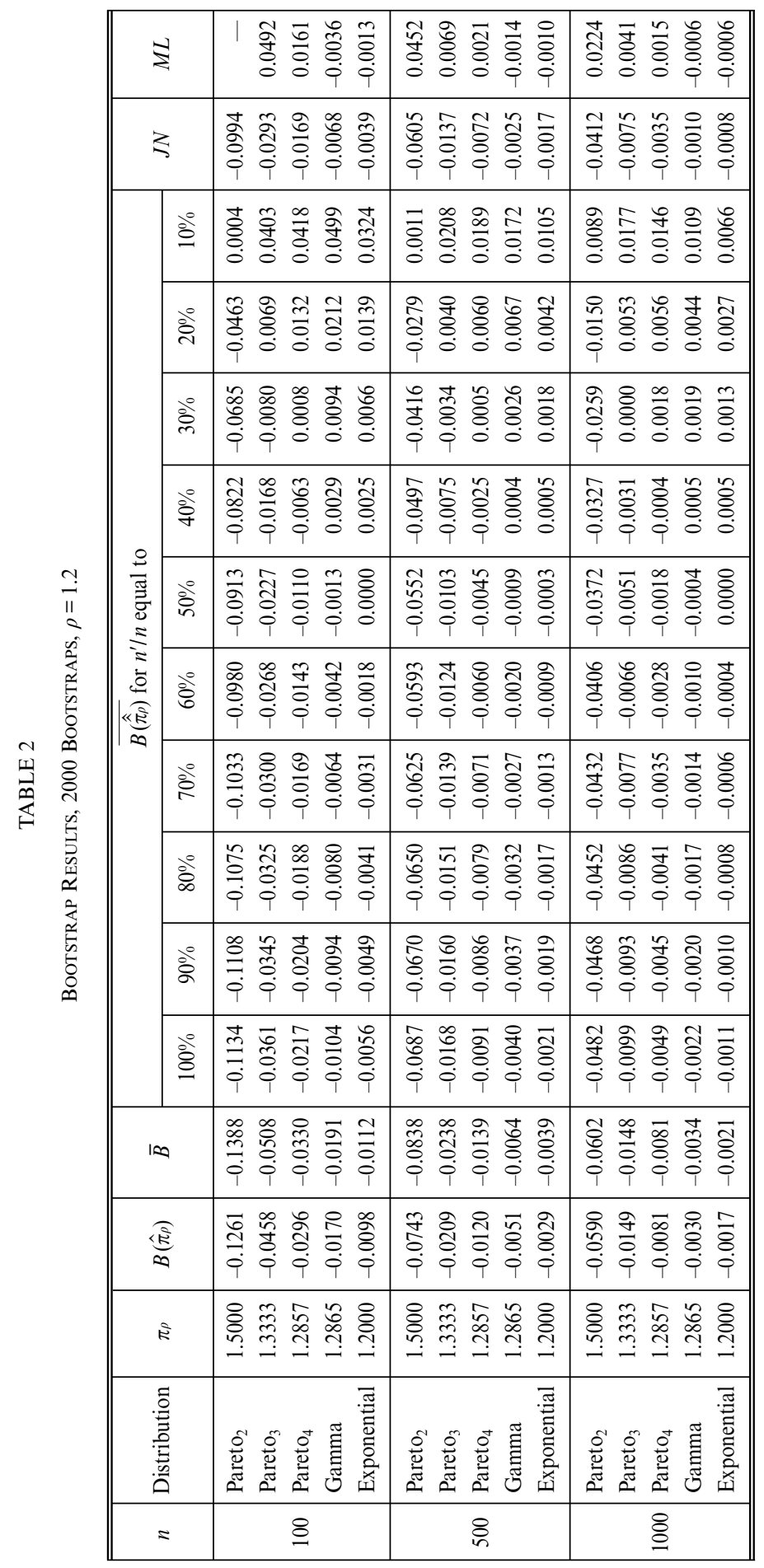




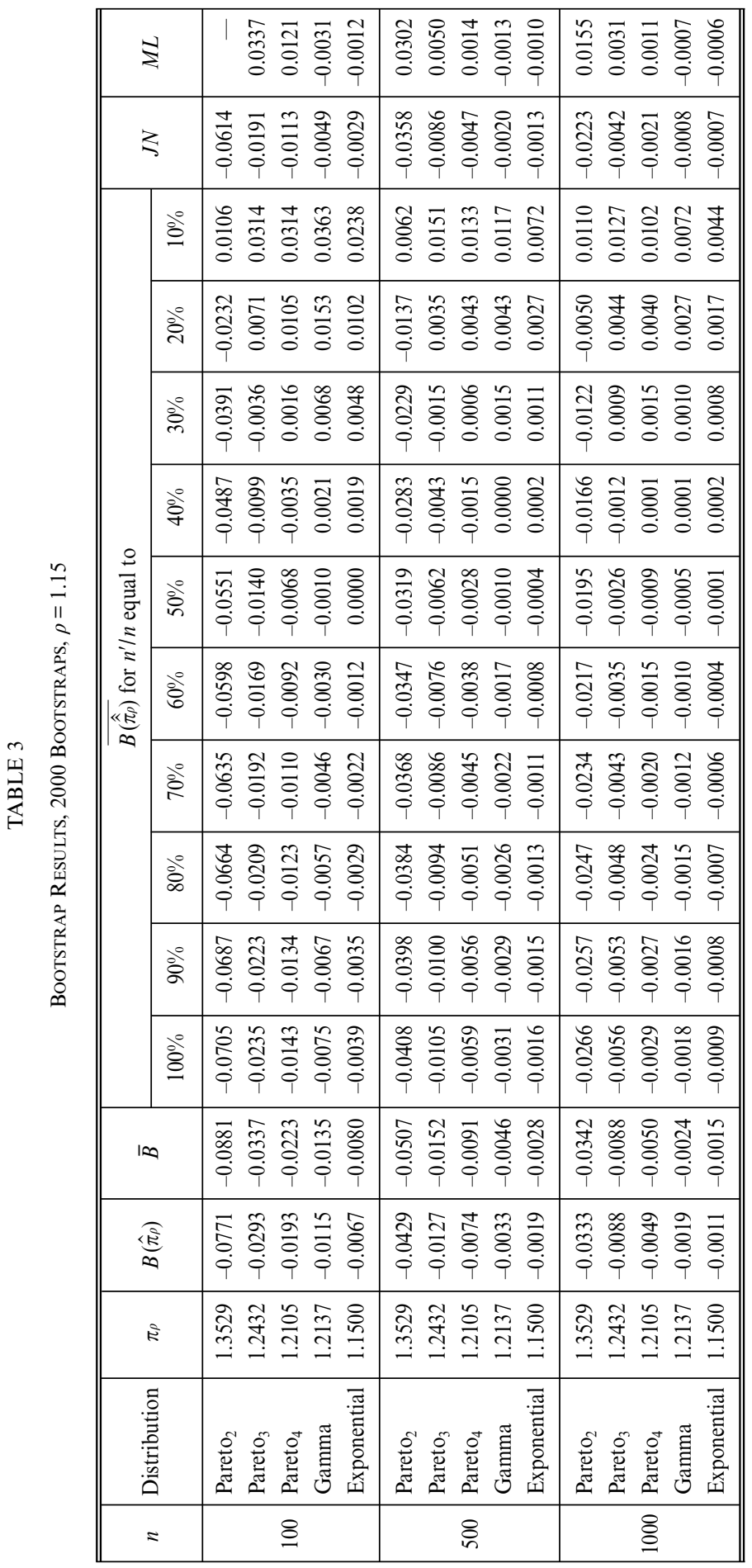




\begin{tabular}{|c|c|c|c|c|c|}
\hline & & & 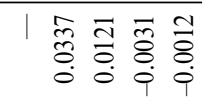 & 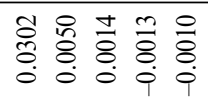 & 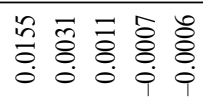 \\
\hline & & & 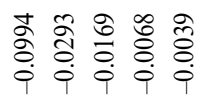 & 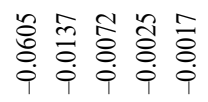 & 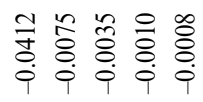 \\
\hline & & ठे & 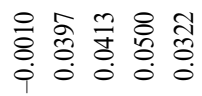 & 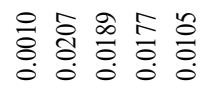 & 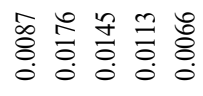 \\
\hline & & ठें & 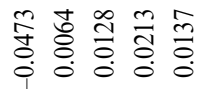 & 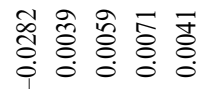 & 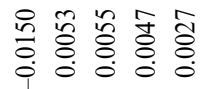 \\
\hline & & ఫ్లి & 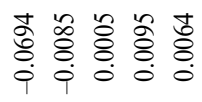 & 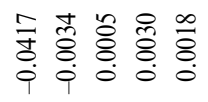 & 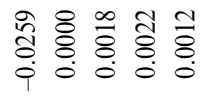 \\
\hline & & ఫें & 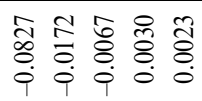 & 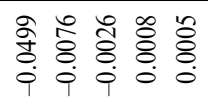 & 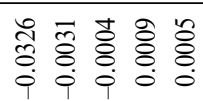 \\
\hline I & 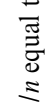 & ठి & 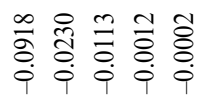 & 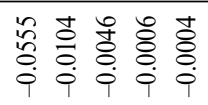 & 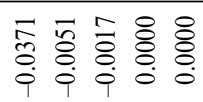 \\
\hline $\begin{array}{l}\frac{\pi}{a} \\
\frac{n}{n} \\
5\end{array}$ & 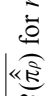 & ठిㅇ & 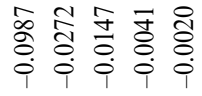 & 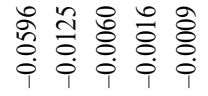 & 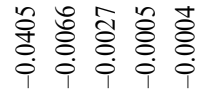 \\
\hline 象 & & ڤ̊ำ & 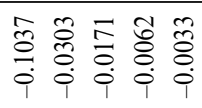 & 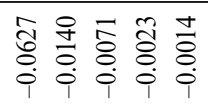 & 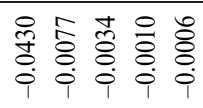 \\
\hline 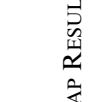 & & ठें & 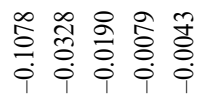 & 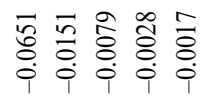 & 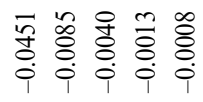 \\
\hline : & & ఫें & 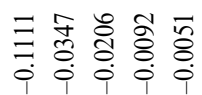 & 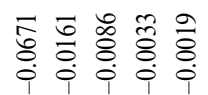 & 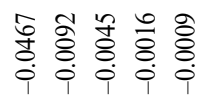 \\
\hline & & தे & 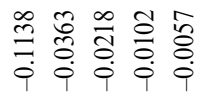 & 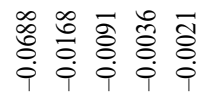 & 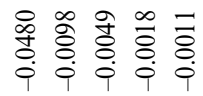 \\
\hline & & & 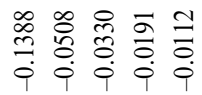 & 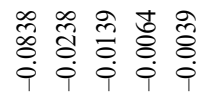 & 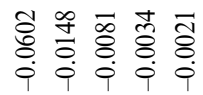 \\
\hline & & & 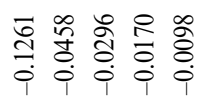 & 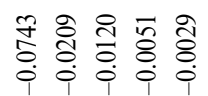 & 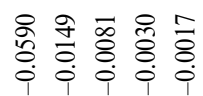 \\
\hline & & $\xi$ & 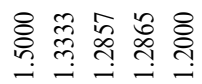 & 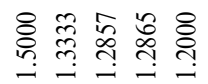 & 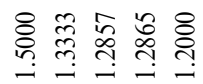 \\
\hline & & & 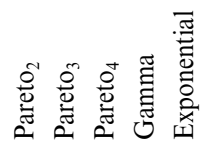 & 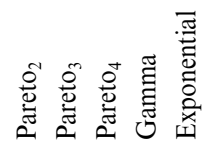 & 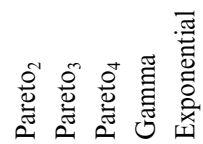 \\
\hline & & $=$ & 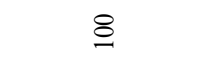 & $\stackrel{8}{8}$ & ¿ \\
\hline
\end{tabular}




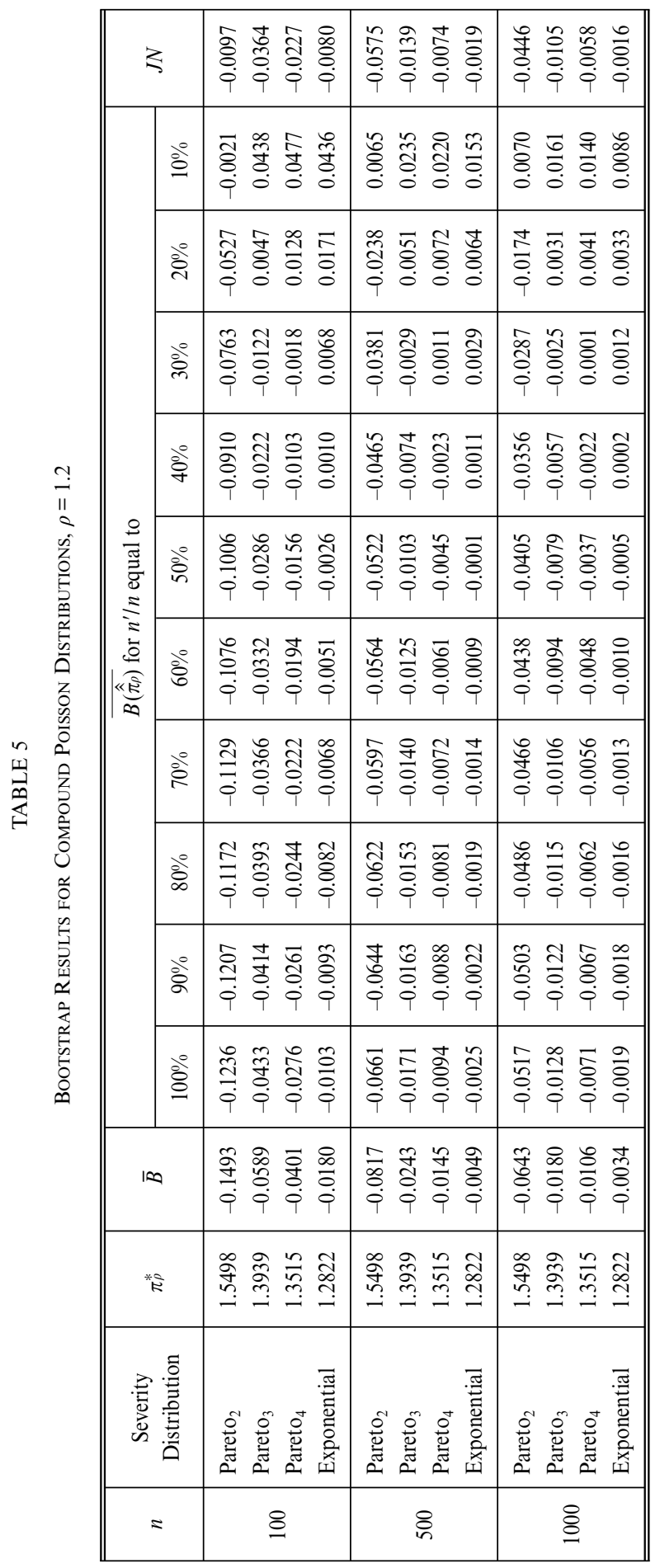




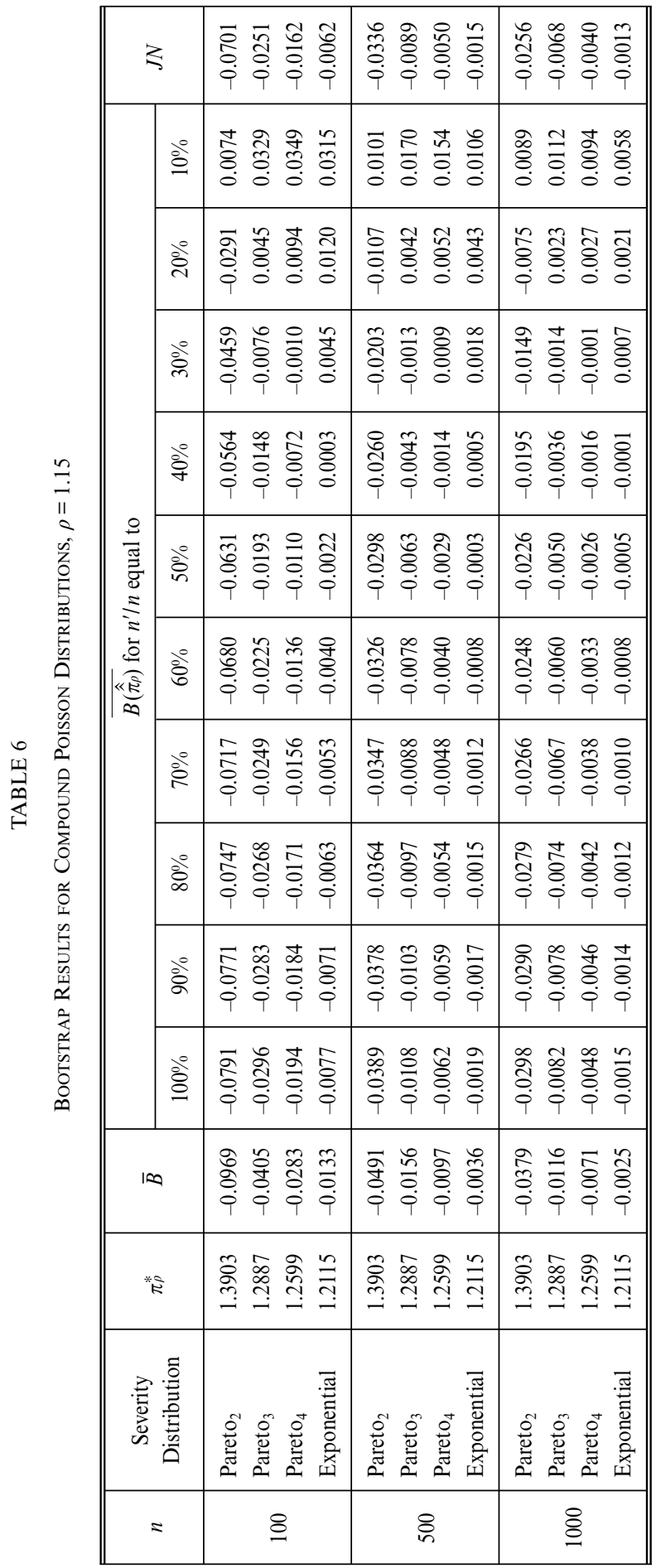




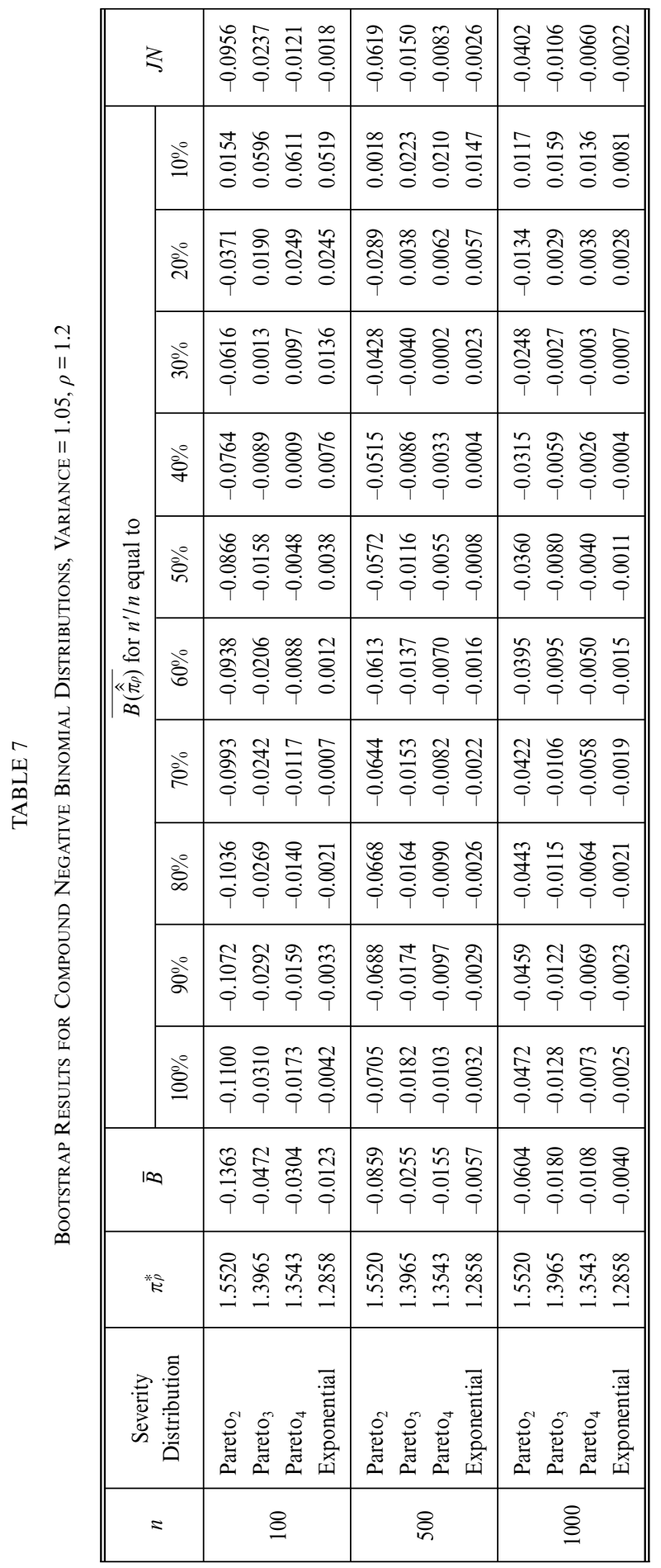




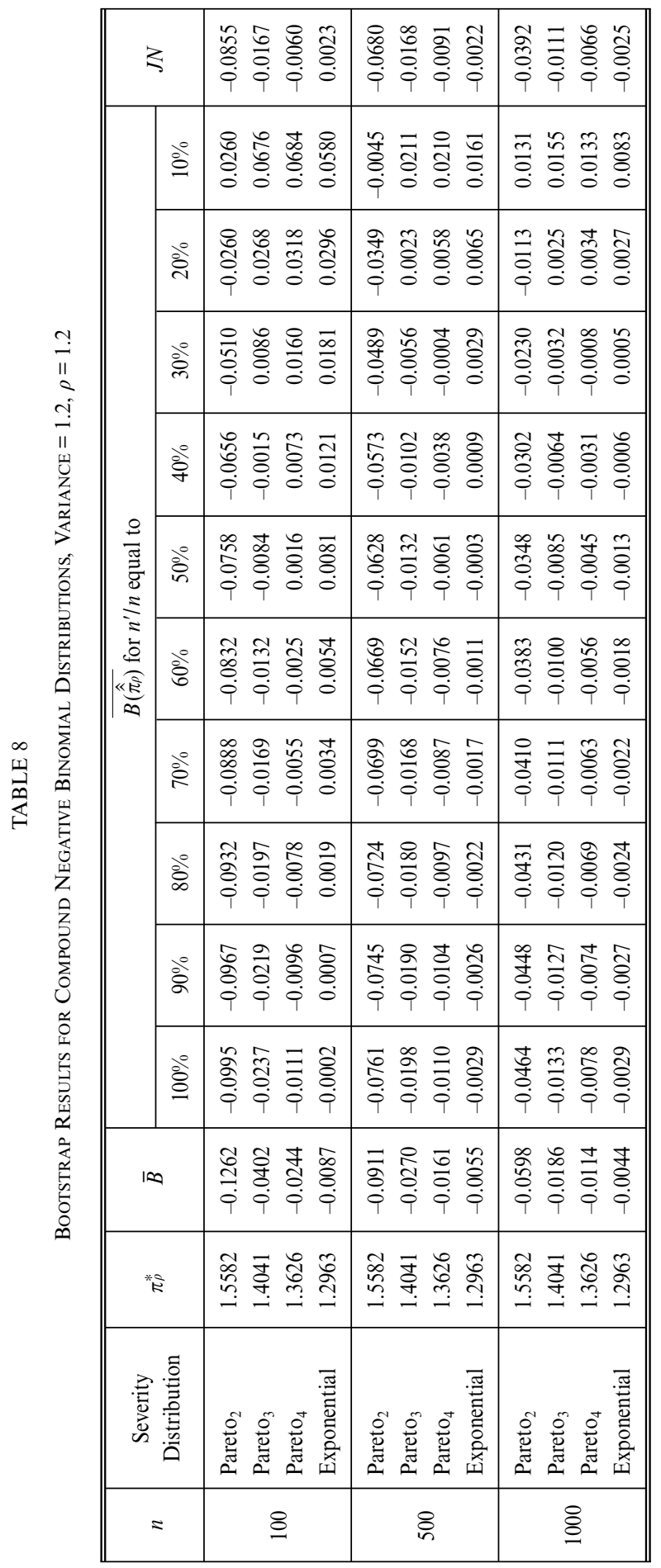

